\title{
ORIGINAL
}

\section{LA INTERVENCIÓN CON ADOLESCENTES Y JÓVENES EN LA PREVENCIÓN Y PROMOCIÓN DE LA SALUD}

Paloma Portero López, Roberta Cirne Lima y Gladys Mathieu

Inner Research. Madrid.

\section{RESUMEN}

Fundamento: A lo largo de los últimos diez años mínimo, se vienen implementando desde diversas instituciones públicas acciones encaminadas a la prevención y promoción de la salud de los jóvenes y adolescentes. El objetivo de este trabajo es evaluar la manera en que las diferentes intervenciones llevadas a cabo por distintas Instituciones Públicas en materia de Prevención y Promoción de la Salud inciden sobre la conducta y prácticas reales de los jóvenes y adolescentes, entre 14 y 25 años, de la Comunidad de Madrid.

Métodos: Se realizó un estudio con metodología estructural o cualitativa utilizando la técnica del grupo de discusión, habiéndose realizado ocho grupos, cuyo discurso se analizó.

Resultados: Los jóvenes, no perciben la existencia de un «sistema» de prevención para la salud al no considerarse objetivo del mismo. Para ellos, la enfermedad, circunscribiéndose al cuerpo-físico, se concibe como momento puntual, transitorio y de fácil resolución a partir de los avances tecnológicos que comporta la medicina actual. Por el contrario, las enfermedades asociadas a la perspectiva psicológica (enfermedad metal disociada del área de lo médico y vinculada al área de lo individual/personal) suscitan interés en tanto que el joven se muestra muy vulnerable frente a ellas.

Conclusiones: Cualquier acción encaminada a generar identificación y, por tanto, la respuesta activa del joven, debe considerar el establecimiento de un vínculo entre el joven y la salud que, no basándose exclusivamente en aportar información, sea capaz de poner en juego un espacio de control del joven respecto de sus prácticas perjudiciales, a la par que señale la autoresponsabilidad de las mismas. Basándonos en ello creemos más adecuado «re-significar»el término prevención con términos cercanos a los de mantener, mejorar o recuperar (en los casos puntuales) la salud, para así connotar la idea de bienestar físico y psíquico.

Palabras clave: Adolescencia. Promoción de la salud. Investigación cualitativa. Percepción de salud.

Correspondencia:

Paloma Portero

Inner Research

Correo electrónico: pportero@inner.es.
ABSTRACT

\section{Measures Aimed at Teenagers and \\ Young Adults Related to Health \\ Promotion and Prevention in the \\ Community of Madrid, Spain}

Background: Over at least the past ten years, measures aimed at health promotion and prevention among young adults and teenagers have been being implemented from different public institutions and, in a certain sense, on a sectorial basis. The overall objective is to assess how the different measures carried out by different Public Institutions with regard to Health Promotion and Prevention have an impact on the actual conduct and practices of teenagers and young adults within the 14-25 age range in the Autonomous Community of Madrid.

Methods: A study based on a structural or qualitative methodology was conducted using the discussion group method, eight groups having been formed and their comments analyzed.

Results: The young adults do not feel any health-related prevention «system» as such exists on not considering themselves to be the object thereof. In the opinion of the young adults, disease, confined to the physical body, is conceived as being a short-lived, temporary and readily remedied based on the technological advances current medicine has to offer. On the contrary, the diseases related to the psychological perspective (mental disease dissociated with the area of the medical and connected to the individual/personal area) arouse interest insomuch as young people are highly vulnerable to these diseases.

Conclusions: Any measure aimed at generating identification and, therefore, the active response of young people must entail creating a connection between young people and health, which, not based exclusively in providing information, is capable of putting a space of control of the youths with regard to their harmful practices into play whilst at the same time highlighting their taking responsibility for their own actions. Considering we believe it best to «redefine» the term «prevention» in terms nearer to those of maintaining, improving or regaining (in short-lived cases) health, to thus connote the idea of physical and mental well-being.

Keywords: Adolescence. Health promotion. Qualitative research. Perception of health. 


\section{INTRODUCCIÓN}

Los modelos de intervención para la promoción y prevención para la salud, en jóvenes y adolescentes, se implementan desde diversas instituciones públicas $\mathrm{y}$, en cierto modo, de forma sectorial en tanto que no existe una reflexión común sobre el tema; es por esto que las acciones encaminadas a dicho fin adolecen de una perspectiva integradora que, hipotéticamente, repercuta en la eficacia de las mismas. La necesidad de dicha reflexión, fue lo que motivó la demanda y posterior realización del estudio cualitativo al que refiere este artículo;

Las diversas investigaciones que se han llevado a cabo en este segmento de la población en relación con la Promoción y Prevención de la Salud, o bien abordan el tema desde una perspectiva particular; es decir en lo relativo a consumos concretos que operan como factor de riesgo ${ }^{1,2}$, en los que si bien la perspectiva de la salud aparece estudiada de forma tangencial respecto del problema central del estudio, ya se apuntan rasgos comunes a los detectados en la investigación que nos ocupa.

En los dos estudios se señala la manera en que los jóvenes incluyen la salud como un significante que si bien se constriñe al ámbito de lo físico, del cuerpo físico-individual, opera como valor constitutivo del vínculo social; en este sentido el estudio sobre el consumo de alcohol posibilita que el «límite» de ingesta del mismo no se establezca en relación con el «otro» ${ }^{1}$ (el grupo de pares) sino que se proponga en el espacio de lo físico (hasta que el cuerpo aguante, podíamos decir parangonando el primero de los estudios). En el mismo orden de cosas, en el estudio de consumo de tabaco ${ }^{2}$ se explicita cómo el concepto de salud en los jóvenes opera como dispositivo capaz de transformar las connotaciones «morales» de vicio, en el caso del consumo de tabaco, por connotaciones de «adicción». Es decir, en ambos se detecta cómo el concepto de salud hace incursiones plagadas de sentido en los espacios/discursos sociales actuales de los jóvenes; la generación de hoy dota a la salud de un espacio simbólico que, si bien se acerca a la modernidad, tiene vocación de ser eje articulador de la idea de calidad de vida y bienestar por todos buscados. En este sentido también cabe destacar un estudio que sobre alimentación en la población escolar habla del necesario equilibrio nutricional como una exigencia a partir de la instauración en el espacio social del discurso de la salud como prevención ${ }^{3}$.

Ahora bien, hasta el momento no se había planteado la cuestión de la salud en los jóvenes desde la perspectiva de la intervención; es decir, la manera en que los discursos y acciones institucionales son dispositivos capaces de modificar pautas de comportamiento que, en definitiva, no son sino expresiones concretas de un discurso social en el que la salud está incluida; fue pues esta perspectiva la que animó a plantear el presente trabajo.

Es por esto, que la demanda de investigación debería abordarse a través de una metodología con capacidad de detectar el valor concedido a la salud por los jóvenes (necesidades, demandas y prácticas concretas), los diversos campos en los que es pertinente introducir el discurso de la salud (vínculo entre sujeto joven y salud) y la particular forma en que debe concretarse dicho discurso (qué deben decir las instituciones sanitarias para producir efectos en los jóvenes). No se trataba, pues, de realizar un análisis de contraste, recuento o forma en que se distribuye las prácticas saludables/ prácticas de riesgo entre la población de estudio (cuantitativo), sino de descubrir el sentido que los jóvenes otorgan a la salud y la manera en que los modelos de intervención institucional se muestran eficaces o con potencial para modificar dicho sentido, incidiendo en su conducta real.

La metodología cualitativa es la única capaz de abordar dicha demanda puesto que fundamentándose en la «palabra» (discurso 
del investigado) permite, mediante el análisis (la interpretación del investigador), dar cuenta del referente social (discurso social) en que se basa lo dicho. La metodología cualitativa se basa en la idea de que la formación discursiva organiza sus campos semánticos — de pertenencia y pertineneciaconforme a un orden social del sentido que no es clasificable en un orden eterno y sustantivo ${ }^{4}$.

Las investigaciones cualitativas adquieren su valor en tanto que actuando como dispositivo conversacional ponen en juego lo «particular» (el habla) siempre en referencia a un discurso genérico (discurso social) ${ }^{5}$.

No se trata, pues, de señalar los espacios de consenso y disenso, ni tampoco de operar en el espacio de las opiniones, sino de encontrar los espacios en los que los sujetos incardinan su decir en el orden de lo social. Se trata pues, de una búsqueda - y captura- del eje articulador de las múltiples posibilidades del decir.

El grupo de discusión, articulación técnica más usual de dicha metodología ${ }^{6-8}$, es la que se muestra con mayor capacidad para extraer el mayor potencial de la misma en la medida que es una propuesta de recogida de información que, por su propia configuración (grupo de personas discutiendo sobre un tema), pone en juego tanto la subjetividad de cada componente como la objetividad del grupo en el que el mismo se inscribe, dando lugar a espacios de interacción y discusión: «simulación de una conversación grupal», que permite articular el sentido social en el puro contraste de los «decires» particulares.

Asegurar, pues la suficiente «dispersión de las hablas particulares» asegura, a la par, la validez de la interpretación. Ahora bien, todo grupo de discusión deberá tener un mínimo de homogeneidad interna de manera tal que no se produzca una inhibición de los sujetos que lo componen: variables discriminativas de composición ${ }^{6,9}$.
A la vez, conformar el grupo en función de dichas variables darán cuenta de la manera en que operan las diferencias entre los distintos segmentos de la población: la distinta forma en que se aborda el problema en estudio o el mayor o menor énfasis en los temas particulares.

Abordar la evaluación de los modelos de intervención utilizados para la Prevención y Promoción de la Salud en los jóvenes, hace necesario conocer previamente lo que la salud representa para el joven, pues solo así podríamos tener un marco de referencia, contexto, que nos permita tanto llevar a cabo la exploración de acciones concretas como proponer alternativas que permitieran optimizar la intervención en dicho segmento de la población.

El objetivo de esta investigación fue la identificación de las principales necesidades y demandas en materia de Prevención y Promoción de la Salud en la población comprendida entre los 14 y 25 años de edad, la identificación del concepto de salud desde la perspectiva de los jóvenes y las variables que entran en juego en dicha percepción, así como conocer las prácticas vinculadas a la salud.

\section{SUJETOS Y MÉTODO}

Se realizaron ocho grupos de discusión entre la población de interés, de dos horas aproximadas de duración cada uno. En la composición general de la muestra se establecieron las siguientes variables: Edad: se consideraron los siguientes intervalos: 14/15 años; 16/17 años; 18/20 años; 21/25 años. Los cortes de edad señalados se deben tanto a motivos técnicos (evitar la inhibición en el grupo) como a intereses concretos de la investigación (referencias en materias de información similares). Sexo: Al igual que ocurriera en el caso de la edad, la discriminación respecto de la participación en los grupos por el sexo de los integrantes, se contemplo en los de menor edad con el fin 
de evitar inhibiciones a la hora de abordar ciertas temáticas. Se realizaron grupos de un solo sexo en los de edades comprendidas entre los 14 y 18 años, convocando grupos mixtos (chicos y chicas al 50\%) en los 4 grupos restantes. Estado socioeconómico: representación al 50\% del total de la muestra de medio bajo y medio-alto (utilizando como baremo los estudios/profesión de los padres). Participación previa en programas de salud: esta variable se aplicó en los segmentos de edad superior discriminando al $50 \%$ de la muestra su participación o no. De la combinación de estas variables se conformó la configuración concreta de trabajo de campo que se muestra en la figura 1 .

Tabla 1

Características de los participantes en cada grupo

\begin{tabular}{|cccccc|}
\hline Grupo & Participantes & Edad & Sexo & $\begin{array}{c}\text { Nivel de estudios del } \\
\text { cabeza de familia }\end{array}$ & $\begin{array}{c}\text { Participación previa en } \\
\text { programas de salud }\end{array}$ \\
\hline GD1 & Adolescentes & $14-15$ años & Varones & medios & Indistinto \\
GD2 & Adolescentes & $14-15$ años & Mujeres & universitarios & Indistinto \\
GD3 & Adolescentes & $16-17$ años & Varones & universitarios & Indistinto \\
GD4 & Adolescentes & $16-17$ años & Mujeres & medios & Indistinto \\
GD5 & Adolescentes & $18-20$ años & Mixto & medio & Participante \\
GD6 & Adolescentes & $18-20$ años & Mixto & universitario & No participante \\
GD7 & Jóvenes & $21-25$ años & Mixto & universitarios & Participante \\
GD8 & Jóvenes & $21-25$ años & Mixto & medios & No participante \\
\hline
\end{tabular}

\section{RESULTADOS}

En primer lugar mostraremos los ejes fundamentales que «rigen» el discurso genérico de los jóvenes, sin señalar, en un primer momento, cómo operan las variables de segmentación utilizadas en la recogida y producción de información. Posteriormente hablaremos de las acciones institucionales más relevantes, la forma en que el concepto de la salud interviene en la comprensión de las diferentes acciones realizadas, así como las demandas concretas de los jóvenes y las vías de optimización en el abordaje de los problemas de salud de los jóvenes.

La concepción de la salud en los jóvenes: La salud para la población estudiada es un factor constitutivo del sujeto joven: naces con salud y sólo el paso del tiempo, la edad, es lo que hace que se vaya deteriorando y agotando. Esta concepción de salud representada a modo de un continuo natural, entre el nacimiento y la muerte, conlleva una correlación inversa entre edad y salud: a menor edad mayor salud y vicecersa. Una persona mayor, aunque tenga salud, no tiene las mismas condiciones que un joven. Cuanto más pasan los años más te tienes que cuidar.

El imaginario (representación ideal) social de que un individuo joven es un individuo sano, unido a la idea de que los avances técnicos de la medicina todo lo pueden, genera a los jóvenes un sentimiento de omnipotencia frente a la enfermedad que procura este alejamiento del discurso institucional sobre la prevención. En este contexto referencial, conciben la enfermedad como un momento puntual y transitorio y, por lo tanto, de fácil resolución. La carencia de salud, así concebida, se percibe más como si de un «accidente» se tratara que de un estado de morbilidad, pues no necesariamente se siente como limitación: Yo tengo diabetes y corro más que mis compañeros». Separados pues, del ámbito de la enfermedad desde el punto de vista médico (genérico), se hace difícil la identificación con el discurso social preventivo dirigidos a ellos. 
Sin embargo, se observa que las enfermedades «sociales», definidas por ellos mismos como aquellas de nueva aparición y muy asociadas a la perspectiva psicológica, enfermedad mental disociada del área de lo médico y unida al espacio de lo individualpersonal, ocupan un espacio central de su discurso, ya que suscitan miedo y peligro que proviene por un lado, de la dificultad que los jóvenes, sea cual fuere su edad y extracción social, tienen para concretar su causa o procedencia: No es lo mismo tener un brazo roto que estar muy mal y no saber por qué. Y por otro, de que la perspectiva psicológica cobra para el joven mucha importancia en su concepción de salud, por la influencia que ejerce el necesario equilibrio de la mente para el bienestar: Una depresión pude causarte incluso problemas físicos.

Es ahí donde, detectándose una falla discursiva del joven que contraviene el discurso general de la supuesta omnipotencia, se señala un espacio de vulnerabilidad para ellos muy relevante: los intereses de los jóvenes en relación con la salud son siempre del orden de lo individual (el problema particular: «mi problema») al igual que lo hiciera la perspectiva psicológica, Y es, tan sólo desde dicha perspectiva dónde el joven pregunta al discurso social, donde éste se muestra insuficiente y, por lo tanto, donde adquiere sentido la información a recibir.

El consumo de drogas y alcohol en los adolescentes (jóvenes a partir de los 16 años) y las relaciones sexuales, uso de preservativos, entre las más jovencitas (chicas de 14 a 15 años) aparece vinculado en este espacio discursivo y por tanto, atrapan la perspectiva individual que lo fundamenta.

\section{Variables y agentes que intervienen en la percepción de la salud y sus practicas}

Edad: Mientras que en los grupos más jóvenes de 14 a 17 años, aparece una vertiente moral que señala cierta culpabilidad en el binomio salud/enfermedad (Yo no he hecho nada malo para que me pase algo) acentuando, en ese sentido, la idea de inmunidad propia del joven en el caso de la salud, en los grupos de edad mayor se acentúa la idea de salud desde la perspectiva del estado de armonía entre cuerpo y psique (La salud es el conjunto de bienestar físico y mental)

Clase social: También se detecta una influencia determinante respecto de la concepción de la salud en relación con el estatus social de pertenencia del joven; así, mientras que en los niveles más bajos poseen un nivel muy precario de información (Yo no sé lo que es el VIH) y trasladando la responsabilidad tanto de la enfermedad cuanto de la información al propio sistema social (Ellos tienen que informarnos más), en los estatus más altos, con mayor nivel de información, cobran mayor peso agentes definidos socialmente y cercanos al círculo de los individuos. En este sentido se destaca el papel de la familia como muy importante tanto en la creación de hábitos (formativos) cuanto de dispositivo de información.

\section{La intervención y sus efectos}

Las intervenciones en materia de Prevención y Promoción para la Salud en los jóvenes son percibidas por éstos a modo de «información generalizada» y altamente vinculada a la enfermedad desde su perspectiva física, alejándose de sus áreas de intereses inmediatos: lo personal. No obstante, se observa cómo los jóvenes delimitan claramente prácticas que favorecen o que perjudican a la salud. Entre las favorecedoras se señalan: la dieta en la alimentación, hacer ejercicio, la higiene personal, el estado anímico (vivir feliz), las relaciones interpersonales (familia y amigos). Entre las perjudiciales destacan: fumar, beber alcohol en exceso, las drogas per se y el estrés. Si las primeras son consecuencia del hábito cotidiano (constancia), las segundas señalan el exceso y la dependencia como una de sus características fundamentales. 
Existe, en este sentido, un reconocimiento explícito de los jóvenes en materia de intervención para la prevención y promoción en materia de salud que se valora positivamente en la medida que se les atribuyen papel informativo fundamental; entre las intervenciones más destacadas figuran las siguientes:

Entre los de menor edad: las charlas en los centros educativos (con personal no docente con el fin de lograr el máximo de desinhibición en las preguntas que susciten), los folletos informativos (es necesario el uso de lenguaje cercano, incluso jerga juvenil para su comprensión (Qué es el coitus interruptus, la marcha atrás), así mismo se demanda su distribución en los centros educativos para fomentar la lectura entre grupos de iguales.

En el grupo de los mayores: Teléfono gratuito informativo (permite la solución anónima de problemas, muy relevante en el grupo de los más mayores)

Los Concursos temáticos $\mathbf{y}$ talleres (muy valorado entre 14 y 18 años) y Campañas televisivas (dispositivo divulgativo masivo y vehículo para dar a conocer programas y acciones existentes).

Respecto de las campañas que aparecen con notoriedad relevante en el conjunto de los grupos realizados hay que destacar aquellas que se valoran positivamente tal cual fueron: «Póntelo, pónselo», «Si te lías úsalo», campaña antidrogas (spots de cocaína, alcohol y pastillas donde el individuo cree vivir algo que en realidad no está ocurriendo). Las valoradas negativamente fueron: «Sal sin ellos»y «A tope sin drogas».

Las valoraciones de uno u otro signo responden a los siguientes parámetros de valoración: Lenguaje: cercano/lejano. Tono: se valora muy positivo el tono coloquial y divertido, por ejemplo: «póntelo, pónselo», campaña antidrogas, gráfica del «si te lías, úsalo», por oposición al autoritario «Sal sin ellos». Las situaciones cotidianas: Campa- ña antidrogas, haciendo el ridículo es altamente valorada en tanto que reconocible por todos los jóvenes como habitual. Mensaje: el valor se produce en la identificación con un sujeto libre, no se generaliza ni culpabili$\mathrm{za}$, por ejemplo «Si te lías úsalo» valorado muy positivamente por el uso del condicional «si», versus «sal sin ellos» valorado muy negativamente ya que culpabiliza la ingesta de alcohol en general. Claridad del mensaje. Por ejemplo «A tope sin drogas» es percibido confusamente ya que muestra un exceso de transgresión propio de las drogas pero sin drogas de lo que se deduce que el joven posee conductas genéricas similares a las de los drogadictos.

\section{DISCUSIÓN}

La información, única característica atribuida por los jóvenes a los modelos hasta ahora utilizados, no basta. Ahora bien, no se debe perder la perspectiva informativa en tanto que posee un gran potencial como dispositivo de apertura a preguntas que bien por vergüenza o por ignorancia el joven no se plantea o elude. Ningún modelo informativo resultará eficaz si no se generan de forma paralela mecanismos de integración que transformen la acumulación de informaciones aisladas, parciales, bien por las temáticas que tratan, bien porque son suscritas por diferentes Instituciones tanto Públicas (Consejerías, Ayuntamientos, Consejos de juventud) como Privadas (Evax, Colgate, etc.) en conocimiento y aprendizaje; es decir, en formación.

No debemos eludir que tanto la vergüenza de preguntar (¿Qué es el pene?) como la ignorancia (No sé por qué ahora todas tenemos quistes de ovarios o problemas en la regla) apuntan, en muchos casos, al desconocimiento y extrañamiento del propio cuerpo.

Es por esto, que si bien no debemos olvidar que la información, cuyo ámbito más relevante se instaura en los amigos (grupo de pares/de referencia: circulación e intercambio de experiencias) y en los medios de co- 
municación (TV y revistas juveniles: fomento de interés por nuevos temas), ocupa un lugar importante como dispositivo que abre la interrogación a nivel individual/personal. Sin embargo, más allá del uso de mecanismos informativos, se percibe la necesidad de intervenir en aquellos espacios que se manifiestan como relevantes respecto del papel claramente formativo y socializador que cumplen en nuestra cultura; tales son la familia y el colegio:

- La familia en la medida que cumple su función socializadora, no sólo trasmitiendo los valores ideológicos vigentes sino porque en dicha transmisión siempre existe una interpretación de las pautas culturales, particular y adaptada a la biografía del hijo; en ese sentido, aparece como un agente social determinante. La armonía en las relaciones familiares son consideradas por los jóvenes como la base de una buena estructura emocional; es decir la perspectiva «psicológica» que ellos mismos se encargan de situar como requisito fundamental del bienestar. (En tus padres siempre puedes confiar»»Siempre vas a confiar mucho en lo que dicen tus padres porque ellos siempre quieren lo mejor para ti.)

- El colegio resulta el espacio más adecuado para intervenir en la prevención y promoción de la salud en la medida que representa una actualización de saber constante y un contexto altamente favorable: lugar donde mayor tiempo pasan y en constante interrelación con el grupo de iguales. A la par, por definición es un espacio de saber en el que la figura del profesor, en tanto que autoridad, representa vicariamente la ley paterna.

Es decir, cualquier modelo de intervención debe considerar y potenciar al máximo la involucración personal del joven que tan sólo se puede conseguir en la incardinación de los agentes mencionados. Para ello sería pertinente diseñar modelos o comunicaciones dirigidos a los padres, una mayor implicación del profesorado en lo referente a intervenciones directas en los Centros.
Las acciones informativas y formativas deben responder a tres demandas de los jóvenes: Coherencia (correlación entre temática e Institución a la vez que unicidad de mensajes). Constancia (mayor frecuencia de actividades y periodicidad predeterminada de las mismas). Extensión (cobertura masiva e inclusión de todas las escuelas y colegios)

Considerando estas dos perspectivas de actuación (involucración del joven e información para el joven), creemos más adecuado «re-significar» el término prevención con términos cercanos a los de mantener, mejorar o recuperar (en los casos puntuales) la salud para así connotar la idea de bienestar físico y psíquico.

También se debe considerar a la hora de la creación de Modelos de intervención en la Prevención y Promoción de la Salud y Campañas Publicitarias que las prácticas saludables del joven están predeterminadas fundamentalmente en función de las variables edad y clase social y que éstas deben ser consideradas en concreto a la hora de cualquier intervención.

En ese sentido, se debe considerar que cumplirá su objetivo (persuadir de la necesidad de informar y formar al joven) considerando los siguientes aspectos:

- Amoldándose a la población diana a la que se dirige: el joven y la familia han de identificarse con ella.

- Informar sobre el problema: Buscar el enfoque y la perspectiva adecuada para plantearlo con eficacia.

- Convencer: el joven tiene responsabilidad su salud.

Respecto al papel que la publicidad cumple dentro de la promoción de la salud entre los jóvenes creemos que, si bien en sí misma no cumple un papel formativo directo, cumple dos funciones colaterales de gran interés a la hora de dotar coherencia en las intervenciones ya que: 
- Por un lado puede funcionar a modo de nexo interno de las partes que configuran las intervenciones concretos: los mensajes deben ser una invitación a la información y deben poseer capacidad para articular los distintos ámbitos en los que se esté trabajando.

- Por otro, puede establecer un vínculo de complicidad con el joven señalando la vocación de la institución en el acercamiento a su problemática particular ${ }^{10}$.

\section{BIBLIOGRAFÍA}

1. Consejería de Salud. La cultura del alcohol entre los jóvenes de la Comunidad de Madrid. Madrid: Consejería de Salud; 1994. Documentos técnicos de salud pública, núm. 9.

2. Consejería de Salud. La cultura del tabaco entre los jóvenes de la Comunidad de Madrid. Madrid: Consejería de Salud; 1993. Documentos técnicos de salud pública, núm. 16 .

3. Consejería de Salud. Factores que determinan el comportamiento alimentario de la población escolar de Comunidad de Madrid. Madrid: Consejería de Salud; 1996. Documentos técnicos de salud pública, núm. 36.
4. Pereña F. Formación discursiva, semántica y psicoanálisis. En: Métodos y técnicas de investigación en Ciencias Sociales. Delgado JM y Gutiérrez J editores. Madrid: Síntesis Psicológica; 1994. p. $465-479$.

5. Ibáñez J. Más allá de la sociología. El Grupo de Discusión: teoría y crítica Madrid: Siglo XXI; 1986

6. Canales M, Peinado A. Grupos de discusión. En: Métodos y técnicas de investigación en ciencias sociales. Madrid: Síntesis psicológica; 1994. p. 287-316.

7. Ibáñez J. Cómo se realiza una investigación mediante grupos de discusión. En: El análisis de la realidad social. Métodos y Técnicas de investigación. Madrid: Alianza Universidad; 1990. p. 489-501.

8. Alonso LE. Entre el pragmatismo y el pansemiologismo. En: La mirada cualitativa en sociología. Madrid: Fundamentos; 1998. p. 57.

9. Jesús Ibáñez: Mas allá de la Sociología. EL Grupo de Discusión: teoría y crítica. Madrid Siglo XXI, 1979.

10. Consejería de Sanidad y Servicios Sociales. Las Representaciones Sociales sobre la salud de los jóvenes madrileños. Documentos Técnicos de Salud Pública n. ${ }^{\circ}$ 45. Madrid: Consejería de Sanidad y Servicios Sociales; 1998. 